\title{
DOSSIER
}

\section{Spécificités de l'élevage de ruminants en montagne}

\section{Avant-propos}

Dans le cadre de l'organisation d'une séance annuelle commune, l'Académie d'Agriculture de France et l'Académie Vétérinaire de France ont choisi pour 2013 le thème de l'élevage en montagne (séance publique du 17 avril 3013).

Sur fond de crise, d'évolution des politiques agricoles européennes et de fluctuation des revenus des éleveurs, l'Académie d'Agriculture, et plus particulièrement la section «Production Animale », réfléchit sur les conditions de restauration de perspectives positives pour l'élevage; elle assume l'hypothèse qu'une clé est l'ancrage territorial de cette activité, associé à un effort d'imagination pour valoriser des produits propres à combler l'attente des citoyens. Choisir en premier lieu d'approfondir l'évolution de l'élevage en montagne et d'en analyser quelques traits spécifiques est un prologue tout indiqué pour cette réflexion.

De manière convergente l'Académie Vétérinaire, confrontée à la déprise territoriale du métier de vétérinaire notamment dans les zones dites difficiles ou défavorisées que représentent certaines régions de montagne, a relevé de manière frappante les risques associés, sur les plans sanitaire et épidémiologique, aux systèmes de transhumance et de mise en commun des troupeaux toutes générations confondues, loin de leurs « camps de base » et des yeux attentifs de leurs propriétaires. Rassembler des troupes issues d'exploitations multiples, c'est aussi favoriser les échanges de leurs agents pathogènes respectifs risquant ainsi d'exposer brutalement à de tels agents des jeunes peu ou pas immunisés. Dans les milieux de montagne la détection précoce des maladies est rendue difficile par la dissémination des animaux sur de vastes territoires.

Loin de toute vision rousseauiste sur la salubrité et la paix des alpages, l'élevage de montagne est de plus confronté à des prédateurs tolérés voire défendus par les profanes (ours et loup) et à la mitoyenneté avec une faune sauvage porteuse d'agents pathogènes non contrôlés, parfois responsables de zoonoses (agent de la brucellose, notamment).

Le thème de l'élevage des ruminants en montagne était donc particulièrement bienvenu pour cette séance commune. L'Editeur d' «Inra Productions Animales », René Baumont, a bien voulu reconnaître l'intérêt des travaux présentés en cette occasion sur quelques aspects géographique, technique, économique, historique et sanitaire, même s'ils ne traitent qu'une partie des problèmes de l'élevage en montagne. Grâce à ses exigences, et à celles des lecteurs critiques des articles, ce dossier a pris figure et pourra constituer une base estimable de références pour de nombreux lecteurs intéressés à l'élevage en montagne.

Dans le respect de la pluralité professionnelle représentée dans nos académies nous avons demandé à de jeunes chercheurs d'une part, à des professionnels praticiens en activité d'autre part, de présenter différentes facettes des forces et faiblesses de ces systèmes pastoraux.

Bruno Martin, avec Michel Lherm et Claude Béranger, dresse un panorama très documenté, dans l'espace et dans le temps, des atouts qui ont permis à des éleveurs dynamiques et imaginatifs de 
maintenir par leurs initiatives et leur sens de l'entraide la stabilité économique de ces modes de production, tout en assurant un service écologique par la préservation de milieux fragiles, qui légitime des soutiens publics spécifiques. Stabilité, il est vrai, à un niveau de revenus moindre que leurs homologues des plaines, beaucoup plus affectés par la crise de l'élevage. L'avenir de la production de ruminants en montagne passe comme en plaine par la nécessaire maîtrise des coûts, mais surtout par la capacité des filières animales à valoriser les atouts spécifiques de ces territoires notamment autour de la notion de paniers de biens.

Marie Dervillé, avec Gilles Allaire, s'interroge sur le devenir de la production laitière de montagne après la suppression en 2015 des quotas laitiers protecteurs. Détaillant les mécanismes de concurrence entre montagne et plaine d'une part, et entre différentes régions de montagne d'autre part, elle montre que la différenciation des productions de montagne par rapport à celles qui peuvent être obtenues en plaine est la stratégie essentielle d'adaptation à la libéralisation des marchés. Développer au niveau d'un territoire une capacité d'innovation et la construction d'une réputation collective, répondant à l'imaginaire des citoyens, conditionnera la survie de la production laitière et fromagère de montagne.

Jean-Pierre Alzieu, en collaboration avec Jeanne Brugère-Picoux et Christophe Brard, montre que les dominantes pathologiques sont d'abord et surtout des maladies dites de groupe, principalement d'étiologie infectieuse. Il explicite les raisons de la plus grande susceptibilité sanitaire de ce type de conduite des troupeaux, face à une diversité de maladies (respiratoires, parasitaires ou boiteries entre autres) qui vont trouver dans le pâturage de montagne des conditions favorables à leur propagation. S'il existe au sein du troupeau un ou plusieurs états cliniques avant la montée à l'estive, ceux-ci seront amplifiés et propagés aux autres animaux réunis, d'où l'importance primordiale des mesures prophylactiques en amont et notamment durant l'hivernage.

Christophe Roy présente, au regard de ces conditions sanitaires particulières, les contraintes spécifiques à l'activité vétérinaire en montagne. Celles-ci contribuent à une légère découverture du maillage vétérinaire. Or, la veille sanitaire est très importante en montagne, sans doute plus qu'ailleurs étant donné les risques liés à l'élevage dans ces régions. L'auteur nous livre quelques pistes d'adaptation afin de maintenir une offre de service aussi efficace et performante que possible alors que l'engouement pour les produits de montagne, notamment fromagers, appelle une vigilance soutenue.

Outre ces avertissements sanitaires, des alertes sont perceptibles : une partie de la production laitière, trop générique, résistera mal à la concurrence des plaines. Des appétits fonciers apparaissent, pas seulement pour des activités de loisir consommatrices de territoires et de ressources en eau : des compétitions nouvelles pour l'accès aux estives apparaissent avec des investisseurs aux capacités financières affirmées.

A un titre ou un autre tous les auteurs de ce dossier soulignent néanmoins les atouts de cette production montagnarde, basée sur une culture collective d'acteurs durs au labeur : recours à une ressource herbagère peu coûteuse, utilisation de races locales rustiques, référence à des terroirs évocateurs de dépaysement, robustesse d'une activité fromagère calée sur des appellations contrôlées et dans laquelle les urbains se plaisent à reconnaître une naturalité respectueuse du bien-être animal, au service du maintien de milieux et de paysages à préserver. La conjonction d'acteurs de montagne combatifs et sobres, de citoyens avides d'images pastorales devrait favoriser l'acceptation de soutiens financiers spécifiques et la pérennisation économique de systèmes d'élevages ancrés sur ces territoires tout en sachant s'adapter.

Jeanne Grosclaude

Vice-présidente de l'Académie d'Agriculture de France
Michel Thibier

Président de l'Académie Vétérinaire de France 